\title{
Satisfacción de los egresados sobre la asesoría de tesis recibida en una Facultad de Salud, Perú
}

\section{Student satisfaction on thesis advising received in a Health School, Perú}

\author{
Oscar Mamani-Benito* \\ Universidad Peruana Unión, Juliaca, Perú \\ ORCID: https://orcid.org/0000-0002-9818-2601 \\ José Ventura-León \\ Universidad Privada del Norte, Lima, Perú \\ ORCID: https://orcid.org/0000-0003-2996-4244 \\ Renzo Felipe Carranza Esteban \\ Universidad San Ignacio de Loyola, Lima, Perú \\ ORCID: https://orcid.org/0000-0002-4086-4845 \\ Madona Tito-Betancur \\ Universidad Tecnológica del Perú, Arequipa, Perú \\ ORCID: https://orcid.org/0000-0002-4611-6899 \\ Christian R. Mejia \\ Universidad Continental, Lima, Perú \\ ORCID: https://orcid.org/0000-0002-5940-7281
}

*Correspondencia:

Email: oscar.mb@upeu.edu.pe
Citar como:

Ventura-León, O., Ventura-León, J., Carranza, R., TitoBetancur, M., \& Mejia, C. (2021). Satisfacción de los egresados sobre la asesoría de tesis recibida en una Facultad de Salud, Perú. Propósitos y Representaciones, 9(3), e1428. http://dx.doi.org/10.20511/pyr2021.v9n3.1428 


\section{Resumen}

Objetivo: Determinar el grado de satisfacción acerca de la asesoría de tesis recibida en egresados de una Facultad de Salud, Perú. Material y métodos: Estudio transversal analitico realizado en 87 egresados, 15 de medicina, 22 de enfermería 19 de nutrición y 31 de psicología, que culminaron su proceso de tesis durante el año 2019. La recolección de datos se realizó mediante un cuestionario de percepción de satisfacción respecto a la asesoría de tesis, previo consentimiento informado. Resultados: en el $86,2 \%$ predomina una insatisfacción respecto a la labor de quien fuera su asesor, $83 \%$ no lo definió como investigador, 93\% opinó que no tenía publicaciones científicas, $93 \%$ que no fue motivado a publicar los resultados de su tesis y $83 \%$ que no recibió apoyo con los trámites. Conclusiones: El grado de satisfacción de los estudiantes sobre la asesoría de tesis fue desfavorable; los asesores no demuestran producción científica y tampoco motivan a publicar los resultados de investigación

Palabras clave: Tesis académica; Mentor; Satisfacción personal.

\section{Summary}

Objective: To determine the degree of satisfaction with the thesis advising received by graduates of a Health School, Peru. Material and methods: Analytical cross-sectional study conducted in 87 graduates, 15 of medicine, 22 of nursing, 19 of nutrition and 31 of psychology, who completed their thesis process during 2019. Data collection was carried out by means of a questionnaire of perception of satisfaction with respect to thesis advising, with prior informed consent. Results: $86.2 \%$ were predominantly dissatisfied with the work of whoever was their advisor, $83 \%$ did not define him/her as a researcher, $93 \%$ felt that he/she did not have scientific publications, $93 \%$ that he/she was not motivated to publish the results of his/her thesis and $83 \%$ that he/she did not receive support with the paperwork. Conclusions: The degree of satisfaction of the students about thesis advising was unfavorable; advisors do not demonstrate scientific production and neither do they motivate to publish research results.

Keywords: Academic thesis; Mentor; Personal satisfaction.

\section{Introducción}

Un análisis del estado de la producción científica en ciencias de la salud entre los años 1996 y 2014, reveló que Latinoamérica había generado el $3.4 \%$ de la producción científica mundial. De este porcentaje, Brasil fue el país con mayor volumen de producción en América del Sur y Perú se ubicó dentro de los países con menor contribución (Carvajal-Tapia \& Carvajal-Rodríguez, 2018).

En este escenario, es propicio incluir en la discusión la figura de las tesis de grado, que, más allá de ser un requisito para obtener un título profesional, representan el principal medio formador para el inicio de la generación de conocimiento científico desde la universidad (Aiquipa et al., 2017).

En ese sentido, la tesis puede definirse como un trabajo académico de elevado rigor científico, cuya elaboración permite desarrollar habilidades básicas para la investigación, tales como el pensamiento crítico y la resolución de problemas a través del método científico (Obuku et al., 2018). Aunque se espera que los productos científicos sean difundidos para su evaluación y posible uso dentro de la comunidad científica (Dhaliwal et al., 2010), desde hace algunos años se evidencia una problemática respecto a su calidad y bajo potencial publicable. Por ejemplo, un estudio en odontología mostró que de 269 tesis solo el $13 \%$ habían sido publicadas en alguna revista científica (Castro-Rodriguez et al., 2018); de igual forma, otro estudio en Psicología 
(Mamani, 2018) halló un $6 \%$ de 149 tesis, y en medicina (Atamari-Anahui, 2015) el $5 \%$ de 398 tesis.

Es a partir de estos hechos que se asume existen limitaciones y serias deficiencias en la elaboración de las tesis de grado, en este caso, mas allá de analizar los contenidos, es necesario enfocar la discusión teniendo como perspectiva la labor de los asesores de tesis desde la percepción de los asesorados. Al respecto, un estudio realizado con médicos recién graduados halló que solo uno de cada diez manifestó estar satisfecho con el rol de su asesor, además, algunos de los factores que influyeron en tal percepción fueron el hecho de que este no sea investigador, y, además no lo incentivara a publicar los resultados de la tesis (Mejia et al., 2016).

Es por lo mencionado en el párrafo anterior que surge la preocupación respecto a la responsabilidad e importancia de las competencias investigativas del asesor de tesis durante el proceso de elaboración del trabajo de grado (Nordsteien \& Horntvedt, 2017), el cual no debería terminar solo en la sustentación, sino, en la publicación de los resultados en revistas científicas (Pasarica et al., 2019). En contraste, la realidad peruana demuestra que los asesores de tesis no cumplen con las expectativas que se espera para impulsar la producción científica estudiantil, en cambio, suelen llegar a ser considerados obstáculos para la culminación del proceso y obtención del título profesional (Castro et al., 2018).

Por todo lo mencionado, es indispensable evaluar el desempeño del asesor de tesis en ciencias de la salud, y ante la falta de investigaciones al respecto, se realizó este trabajo con el objetivo de determinar el grado de satisfacción acerca de la asesoría de tesis recibida en egresados de una Facultad de Salud, Perú.

\section{Método}

\section{Diseño}

El estudio corresponde a un diseño transversal analitico (Quispe et al., 2020).

\section{Participantes}

Participaron voluntariamente un total de 87 egresados provenientes de los programas de medicina, enfermería, nutrición y psicología $(15,22,19$ y 31) quienes lograron culminar su proceso de tesis en el año 2019. Esta cantidad se logró determinar en base a lo suscrito en las actas de sustentación provistas en la secretaria académica de la escuela profesional y facultad. Por ello, se tomó un muestreo no aleatorio de tipo censal incluyendo a la totalidad de egresados.

\section{Instrumentos}

La recolección de datos se hizo por medio de un cuestionario de percepción de satisfacción respecto a la asesoría de tesis, proveniente de un estudio similar (Mejía et al., 2016). Esta consta de ocho preguntas cerradas con opciones de respuesta dicotómica (si, no) que tienen el propósito de medir el grado de satisfacción del tesista en relación a la labor de su asesor de tesis. Para confirmar su idoneidad en el presente estudio, ya que el cuestionario se utilizó solo con población de egresados de medicina, se procedió a corroborar la validez basada en el contenido (VenturaLeón, 2019). En este caso, participaron siete docentes ( 2 de medicina, 2 de enfermería, 2 de psicología y 1 de nutrición) asesores de tesis con publicaciones científicas, quienes evaluaron la claridad, relevancia y representatividad de las preguntas. Una vez obtenida las calificaciones se procedió a calcular el coeficiente $\mathrm{V}$ de Aiken, resultando un valor de .95 el cual esta por encima del corte .80 que es indicativo de alta validez. Así mismo, en la presente investigación, el grado de consistencia interna del cuestionario fue evaluado mediante el coeficiente Kudar-Richardson, obteniendo un valor de .91 , que es indicativo de alta fiabilidad. 


\section{Procedimientos}

El cuestionario se trasladó a un formulario electrónico a través de Google Forms. Para el reclutamiento se contactó a los egresados vía correo electrónico, WhatsApp y Facebook, teniendo como tiempo límite para la espera de respuestas, de 15 días. A cada participante se le envió una invitación para participar de una encuesta virtual, explicando en la primera parte el propósito y objetivo del estudio; seguido de la solicitud del consentimiento informado, en la que se enfatizó que la información brindada sería utilizada solo para fines de investigación y que el manejo de los datos seria completamente confidencial.

\section{Análisis de datos}

En el procesamiento de datos se utilizó estadística descriptiva; para las variables categóricas se halló las frecuencias y porcentajes y para las cuantitativas la mediana y rango intercuartílico. Luego se obtuvo las asociaciones de cada una de las preguntas según el sexo (se utilizó las pruebas estadísticas del chi cuadrado y la exacta de Fisher, según corresponda) y la edad (en todos los casos se usó la prueba estadística de la suma de rangos). Se consideró al .05 como el límite de la significancia estadística.

\section{Consideraciones éticas}

El estudio fue revisado y aprobado por el comité de ética de la Dirección Regional de Salud de Puno, jurisdicción donde se encuentra la faculta de salud estudiada.

\section{Resultados}

Según la Tabla 1, se observa en mayor proporción que el 63.2\% (55) fueron mujeres, siendo la mediana de edades 26 años (rangos intercuartílicos: 24-27 años).

Tabla 1.

Características del sexo y edad de los egresados de salud

\begin{tabular}{lcccc}
\hline Sexo & Frecuencia & Porcentaje & Mediana de edad & Rangos intecuartílicos \\
\hline Hombres & 32 & $36.8 \%$ & & \\
Mujeres & 55 & $63.2 \%$ & 26 & $24-27$ \\
Total & 87 & $100.0 \%$ & & \\
\hline
\end{tabular}

Según la Tabla 2 existen mayores porcentajes en el grupo que demuestra estar insatisfecho con la labor de su asesor, el $82.7 \%$ no lo define como investigador, el $93.3 \%$ reconoce que no ha publicado un artículo científico, el $93.3 \%$ que no fue motivado a publicar la tesis y $82.7 \%$ que no recibió apoyo con los trámites.

Tomando los puntos más positivos, $69.3 \%$ manifiesta que les permitieron elegir su asesor, $50.7 \%$ refiere que su asesor demuestra experiencia en asesoría de tesis y $68.0 \%$ que fue motivado respecto al desarrollo del trabajo. 
Tabla 2.

Grado de satisfacción respecto a la asesoría de tesis recibida en los egresados de salud

\begin{tabular}{lccccc}
\hline \multirow{2}{*}{ El asesorado } & & \multicolumn{4}{c}{ Satisfacción } \\
\cline { 3 - 6 } & & \multicolumn{3}{c}{ Satisfecho } & Insatisfecho \\
\hline Lo definiría como investigador & $\mathrm{Si}$ & 1 & $8.3 \%$ & 13 & $17.3 \%$ \\
Ha publicado un artículo científico & $\mathrm{No}$ & 11 & $91.7 \%$ & 62 & $82.7 \%$ \\
& $\mathrm{Si}$ & 1 & $8.3 \%$ & 5 & $6.7 \%$ \\
Yo lo elegí como mi asesor & $\mathrm{No}$ & 11 & $91.7 \%$ & 70 & $93.3 \%$ \\
& $\mathrm{Si}$ & 8 & $66.7 \%$ & 52 & $69.3 \%$ \\
Tiene experiencia en asesoría de tesis & $\mathrm{No}$ & 4 & $33.3 \%$ & 23 & $30.7 \%$ \\
& $\mathrm{Si}$ & 7 & $58.3 \%$ & 38 & $50.7 \%$ \\
Me motivó en el proceso de tesis & $\mathrm{No}$ & 5 & $41.7 \%$ & 37 & $49.3 \%$ \\
& $\mathrm{Si}$ & 7 & $58.3 \%$ & 51 & $68.0 \%$ \\
Me incentivo a publicar la tesis & $\mathrm{No}$ & 5 & $41.7 \%$ & 24 & $32.0 \%$ \\
Recibí su apoyo en los tramites & $\mathrm{Si}$ & 0 & $0.0 \%$ & 5 & $6.7 \%$ \\
& $\mathrm{No}$ & 12 & $100.0 \%$ & 70 & $93.3 \%$ \\
& $\mathrm{Si}$ & 3 & $25.0 \%$ & 13 & $17.3 \%$ \\
& $\mathrm{No}$ & 9 & $75.0 \%$ & 62 & $82.7 \%$ \\
\hline
\end{tabular}

En cuanto a los resultados del análisis bivariado, la Tabla 3 muestra la satisfacción según el sexo y la edad. Los valores p fueron obtenidos con la prueba exacta de Fisher, en los valores descriptivos de la edad se muestran las medianas (rangos intercuartílicos); por lo tanto, resultó estadísticamente diferente el sexo según la insatisfacción de la asesoría, ya que, las mujeres estaban insatisfechas en un $7.3 \%(n=4)$ y los hombres en un $25.0 \%(\mathrm{n}=8)$ (valor $\mathrm{p}=.002$, obtenido con la prueba estadística del chi cuadrado). Por otra parte, aquellos que percibían que sus asesores no tenían experiencia asesorando fueron más jóvenes (mediana: 24 años; rango intercuartílico: 23-26 años) en comparación de los que percibían que si (mediana: 26 años; rango intercuartílico: 24-28 años) (valor $\mathrm{p}=.024$, obtenido con la prueba estadística de la suma de rangos).

Tabla 3.

Sexo y edad asociados a la insatisfacción respecto a la asesoría de tesis recibida en los egresados de salud

\begin{tabular}{|c|c|c|c|c|c|c|}
\hline \multirow{2}{*}{ Preguntas } & \multicolumn{2}{|c|}{ Sexo } & \multirow{2}{*}{$\begin{array}{l}\text { Valor } \\
\text { p* }^{*}\end{array}$} & \multicolumn{2}{|c|}{ Edades } & \multirow{2}{*}{$\begin{array}{l}\text { Valor } \\
\mathrm{p}^{* *}\end{array}$} \\
\hline & Mujeres & Varones & & No & $\mathrm{Si}$ & \\
\hline $\begin{array}{l}\text { ¿Insatisfecho con la } \\
\text { asesoría? }\end{array}$ & $4(7.3 \%)$ & $8(25.0 \%)$ & .021 & $\begin{array}{c}26 \\
(24-27)\end{array}$ & $\begin{array}{c}26 \\
(22-27)\end{array}$ & .784 \\
\hline $\begin{array}{l}\text { Lo definiría como } \\
\text { investigador }\end{array}$ & $8(14.6 \%)$ & $6(18.8 \%)$ & .607 & $\begin{array}{c}26 \\
(24-27)\end{array}$ & $\begin{array}{c}24 \\
(21-26)\end{array}$ & .075 \\
\hline $\begin{array}{l}\text { ¿Ha publicado un } \\
\text { artículo científico? }\end{array}$ & $5(9.1 \%)$ & $1(3.1 \%)$ & $.407 *$ & $\begin{array}{c}26 \\
(24-27)\end{array}$ & $\begin{array}{c}24 \\
(21-26)\end{array}$ & .225 \\
\hline $\begin{array}{l}\text { Yo lo elegí como mi } \\
\text { asesor }\end{array}$ & $37(67.3 \%)$ & $23(71.9 \%)$ & .655 & $\begin{array}{c}24 \\
(24-26)\end{array}$ & $\begin{array}{c}26 \\
(23-28)\end{array}$ & .211 \\
\hline $\begin{array}{l}\text { ¿Tiene experiencia en } \\
\text { asesoría de tesis? }\end{array}$ & $27(49.1 \%)$ & $18(56.3 \%)$ & .519 & $\begin{array}{c}24 \\
(23-26)\end{array}$ & $\begin{array}{c}26 \\
(24-28)\end{array}$ & .024 \\
\hline $\begin{array}{l}\text { ¿Me motivó en el } \\
\text { proceso de tesis? }\end{array}$ & $35(63.6 \%)$ & $23(71.9 \%)$ & .432 & $\begin{array}{c}26 \\
(24-26)\end{array}$ & $\begin{array}{c}26 \\
(23-27)\end{array}$ & .916 \\
\hline $\begin{array}{l}\text { ¿Me incentivo a } \\
\text { publicar la tesis? }\end{array}$ & $3(5.5 \%)$ & $2(6.3 \%)$ & $1.000 *$ & $\begin{array}{c}26 \\
(24-27)\end{array}$ & $\begin{array}{c}26 \\
(24-27)\end{array}$ & .760 \\
\hline $\begin{array}{l}\text { ¿Recibí su apoyo en los } \\
\text { trámites? }\end{array}$ & $9(16.4 \%)$ & $7(21.9 \%)$ & .522 & $\begin{array}{c}26 \\
(24-27)\end{array}$ & $\begin{array}{c}26 \\
(22-27)\end{array}$ & .649 \\
\hline
\end{tabular}

Nota: *Chi cuadrado; ** Suma de rangos 


\section{Discusión}

La necesidad de generar mayor producción cientifica en ciencias de la salud en el ámbito peruano (Mamani-Benito, 2021a), motiva a los investigadores a indagar sobre aspectos especificos del proceso de elaboración de las tesis. En este caso, se sabe que un producto científico de calidad y con potencial publicable es resultado de la motivación del tesista y las capacidades del asesor (Corrales-Reyes et al., 2018), quien no solo debería brindar orientación técnica, mas bien apoyo y supervisión en las diversas etapas de su elaboración. Ante ello, se tuvo el objetivo de determinar el grado de satisfacción acerca de la asesoría de tesis recibida en egresados de una Facultad de Salud en el Perú.

Uno de los primeros hallazgos tiene que ver con los porcentajes donde los egresados se muestran insatisfechos con la labor de su asesor. Esto coincide con lo encontrado en un estudio similar (Mejia et al., 2016) donde cerca del 90\% demostró estar insatisfecho con el asesoramiento recibido. Aunado a esto, la percepción de que a su asesor le falta experiencia en investigación, no sea considerado investigador y tampoco tenga experiencia en publicación científica, concuerda con lo hallado en otros estudios (Atamari-Anahui et al., 2015; Atamari-Anahui et al., 2016), por lo que este hecho se hace una constante en algunas facultades de ciencias de la salud en Perú.

Por otro lado, el hecho de que los participantes perciban que no fueron motivados a publicar la tesis a pesar de haber logrado sustentarla satisfactoriamente, contrasta con la percepción de otro grupo que si refiere haber sido motivado, pero, en relación al cumplimiento y finalización del proceso, por lo tanto, esto es evidencia de que la motivación es un aspecto central en la investigación formativa, pues docentes motivados, cordiales y dignos de confianza influyen positivamente en el ejercicio de la investigación en el pregrado (Hernandez et al., 2016).

En la misma línea, a pesar de que la publicación de la tesis sería la mejor prueba para evidenciar la calidad del mismo, según la percepción de los egresados los asesores de tesis no enfocan su labor en tal sentido (Sipahi et al., 2012). Tampoco se observa el apoyo con el proceso de documentación, por lo que esto tendría una implicancia respecto a la gestión de la universidad en cuestión de los procesos de investigación, pues en este caso, lo ideal sería que los docentes que asumen la responsabilidad de asesorar tengan pleno conocimiento de los requisitos a presentar en cada etapa del proceso de investigación. Asi, no se espera solamente que el asesor mantenga una relación directa y cercana con el estudiante, sino, que pueda asegurar la calidad del trabajo aún en cuestión de los procedimientos (Aiquipa et al, 2017).

Otro hallazgo importante es el hecho de que un gran porcentaje reconozca que se les permitió elegir a su asesor, y esto haya tenido un impacto favorable sobre el desarrollo de la tesis (Alarco et al. 2010); no obstante, cuando no se elige al asesor, existe la posibilidad que el tesista busque asesorías privadas fuera de las aulas universitarias (Moreno-Loaiza, 2013). Por lo tanto, se resalta la labor del asesor como responsable no solo de la revisión crítica, redacción y aprobación del informe final de investigación (Díaz-Vélez et al., 2019), sino, también en la gestión que implica su aprobación (Santa Cruz \& Duran, 2018).

Los hallazgos de esta investigación difieren de otros estudios. Por ejemplo, con el trabajo realizado por Hernández et al. (2016), que a diferencia del presente, contó con la participación de egresados de posgrado, lo cual supone que una formación a tal nivel provee de docentes con mayor experiencia y trayectoria en investigación (Casimiro et al., 2020). Así también, con la población utilizada por Mejia et al. (2016), que estuvo conformada solo por médicos recién egresados y titulados, en cambio, el presente estudio incluyó egresados de otras carreas de salud. De igual forma, con otro trabajo de Mejia et al. (2017), aunque con diferente perspectiva, donde se halló que los estudiantes de medicina se mostraban satisfechos respecto a la enseñanza de cursos de ciencias básicas. En consecuencia, es importante recalcar que en el Perú el campo de la medicina es uno de los mas investigados en cuestión de procesos de investigación y también es el 
que genera mayor número de publicaciones en comparación a otras áreas (Gonzales-Saldaña et al., 2018).

Por lo presentado, se asume que los resultados expuestos tienen implicancias sobre el estudio de la calidad de los procesos de investigación en el pregrado peruano, especialmente sobre el desempeño que demuestra el cuerpo de asesores de trabajos de grado. Asi, se puede asumir que la experiencia en publicación, calidad de orientación y acompañamiento por parte del asesor influyen directamente sobre la motivación por la investigación en los tesistas (Veytia \& Contreras, 2019), incluso en la desiciòn de querer publicar los resultados en una revista científica (Bastidas \& Benites, 2016). De esta manera, las facultades de ciencias de la salud deberían contar con recursos humanos calificados para la investigación, con ello asegurar el desarrollo del conocimiento científico y tecnologico en el ámbito de la salud (Cheol et al., 2020). Desafio aun imperante en el Perú, ya que reportes iniciales dan cuenta de que incluso las universidades que lideran los rankings no aseguran que los productos científicos se difundan a travez de publicaciones periodicas (Mamani-Benito et al., 2021b).

La presente investigación tiene algunas limitaciones. La principal es el tamaño de la muestra, pues esta no permite generalizar los hallazgos al contexto de la totalidad de egresados en el Perú. Por otro lado, el método de recolección de datos fue la encuesta, que es sabido que instrumentos de autoinforme presentan algunas limitaciones al momento de explorar algunos fenómenos como es la percepción de la satisfacción en relación a la labor del asesor de tesis. En consecuencia, se recomienda replicar la investigación incluyendo egresados de universidades de otras regiones del Perú, además, incluir un método de recolección como la entrevista estructurada y la evaluación mediante grupos focales.

En conclusión, predomina una insatisfacción respecto a la labor del asesor de tesis, según la percepción de egresados de una Facultad de Ciencias de la Salud peruana; además, se refiere que los asesores no demuestran producción científica y tampoco motivan a sus asesorados a publicar los resultados de la investigación. Esto, perjudica la intención de que los tesistas orienten sus investigaciones hacia la difusión de los resultados en una revista de especialidad.

\section{Referencias}

Aiquipa, J., Ramos, C., Curay, R., Guizado, L. (2017). Factores implicados para realizar o no realizar tesis en estudiantes de psicología. Propós Represent, 6(1), 21-52. http://dx.doi.org/10.20511/pyr2018.v6n1.180

Alarco, J., Aguirre-Cuadros, E., Aliaga-Chávez, Y., Álvarez-Andrade, E. (2010). Factores asociados a la realización de tesis en pregrado de Medicina en una universidad pública del Perú. CIMEL,15(2), 66-70. https://www.cimel.felsocem.net/index.php/CIMEL/article/view/137

Atamari-Anahui, N., Roque-Roque, J., Robles-Mendoza, R., Nina, P., Falcón-Huancahuiri, B. (2015). Publicación de tesis de pregrado en una facultad de Medicina en Cusco, Perú. Rev Med Hered, 26(4), 217-221. http://www.scielo.org.pe/scielo.php?script=sci_arttext\&pid=S1018130X2015000400003

Atamari-Anahui, N., Sucasaca-Rodríguez, C., Marroquin-Santa, J. (2016). Publicación científica de asesores de tesis de pregrado en una escuela de medicina de Cusco, Perú. Investigación Educ Médica, 5(20), 279-80. https://dx.doi.org/10.1016/j.riem.2016.05.002

Bastidas, M., \& Benites, R. (2016). Incidencia de la motivación en la producción científica institucional.

$$
\text { Retos, 11(1), }
$$

65-87. https://retos.ups.edu.ec/index.php/retos/article/view/11.2016.05/1097

Carvajal-Tapia, A., Carvajal-Rodríguez, E. (2018). Status of scientific production in Medicine in South America. 1996-2016. Rev Fac Med, 66(4), 595-600. https://doi.org/10.15446/revfacmed.v66n4.67215 
Castro, Y., Cósar-Quiroz, J., Arredondo-Sierralta, T., Sihuay-Torres, K. (2018). Scientific production of theses supported and published by students of Dentistry. Educ Médica, 19, 85-9. https://doi.org/10.1016/j.edumed.2017.04.002

Castro, Y., Sihuay-Torres, K., Perez-Jiménez, V. (2018). Scientific production and perception of research by students of dentistry. Educ Medica, 19(1), 19-22. https://dx.doi.org/10.1016/j.edumed.2016.11.001

Casimiro, W.H., Casimiro, C.N., \& Casimiro, J.F. (2020). Los posgrados y la investigación científica en las universidades peruanas. Rev. Mendive, 18(1), 155-169. http://scielo.sld.cu/scielo.php?script=sci_arttext\&pid=S1815-76962020000100155

Corrales-Reyes, I.E., Reyes-Perez, J.J., \& Diaz-Santoya, M.L. (2018). Asesores de tesis y publicación de artículoscientíficos en el pregrado. Rev Med Hered, 29, 201-202. http://www.scielo.org.pe/pdf/rmh/v29n4/a11v29n4.pdf

Cheol, J., Li, X., Byun, B., \& Nam, I. (2020). Creación de un sistema de coordinación de los recursos humanos, la investigación y la industria para el desarrollo económico impulsado por el conocimiento y la tecnología en el sur de Asia. Revista Internacional de Desarrollo Educativo, 74, 102161. https://doi.org/10.1016/j.ijedudev.2020.102161

Dhaliwal, U., Singh, N., Bhatia, A. (2010). Masters theses from a university medical college: Publication in indexed scientific journals. Indian $J$ Ophthalmol, 58(2), 101. https://doi.org/10.4103/0301-4738.60070

Díaz-Vélez, C., Fernández-Mogollón, J., Apolaya-Segura, M., Pisfil-Farroñay, Y. (2019). Thesis advisers: Do they meet criteria for authorship? Cad Saude Publica, 35(2), 75-82. https://doi.org/10.1590/0102-311x00251318

Gonzales-Saldaña, J., Chavez-Uceda, T., Lemus-Arteaga, K., Silva-Ocas, I., Galvez-Olortegui, T., Galvez-Olortegui, J. (2018). Scientific production of the medical faculty of a Peruvian university in SCOPUS and PubMed. Educ Médica, 19, 128-34. https://doi.org/10.1016/j.edumed.2017.01.010

Hernández, C., Jiménez, M., Guadarrama, E., Rivera, Á. (2016). La percepción de la motivación y satisfacción de la tutoría recibida en estudios de posgrado. Form Univ, 9(2), 49-59. https://scielo.conicyt.cl/pdf/formuniv/v9n2/art06.pdf

Mamani, O. (2018). Calidad metodológica y características de las tesis de pregrado de psicología de una universidad privada del Perú. Propós Represent;6(2), 301-311. http://dx.doi.org/10.20511/pyr2018.v6n2.224

Mamani-Benito, O. (2021a). Rasgo conciencia de la personalidad: factor determinante en la producción científica peruana. Educación Médica, 22, S237. https://www.elsevier.es/esrevista-educacion-medica-71-pdf-S1575181320301819

Mamani-Benito, O., Tito-Betancur, M., Rodriguez-Alarcon, F., \& Mejia, C. (2021b). ¿Se publican las tesis sustentadas de psicología en el Perú? Un análisis de tres universidades mejor posicionadas en el ranking Scimago. Propos Represent, 29(2), e1202. http://dx.doi.org/10.20511/pyr2021.v9n2.1202

Moreno-Loaiza, O., Mamani-Quispe, P., \& Mayta-Tristan, P. (2013). Compra y venta de tesis online: Un problema ético por controlar. Rev Peru Med Exp Salud Publica, 30(2), 352354. https://doi.org/10.17843/rpmesp.2013.302.222

Mejia, C., Cáceres, O., Vera, A., Inga-Berrospi, F., Mayta-Tristán, P. (2016). Percepción y factores asociados a insatisfacción que los médicos recién graduados tienen de sus asesores de tesis, Lima-Perú. Rev Cuba Educ Medica Super,30(4), 340-8. http://scielo.sld.cu/scielo.php?script=sci_arttext\&pid=S0864-21412016000400010

Mejia, C., Verastegui-Diaz, A., Aranzabal-Alegria, G., Quiñonez-Laveriano, D., Lopez, C., ToroHuamanchumo, C., Astocondor-Altamirano, J., Arce-Villalobos, L., \& Diaz, S. (2017). Nivel de satisfacción de ciencias básicas en estudiantes de medicina de siete universidades de Perú. Educ Med Super, 31(3), 45-52. http://scielo.sld.cu/scielo.php?script=sci_arttext\&pid=S0864-21412017000300006

Nordsteien, A., Horntvedt, M., Syse, J. (2017). Use of research in undergraduate nursing students' theses: A mixed methods study. Nurse Educ Today, 56, 23-8. https://doi.org/10.1016/j.nedt.2017.06.001 
Obuku, E., Lavis, J., Kinengyere, A., Ssenono, R., Ocan, M., Mafigiri, D. (2018). A systematic review on academic research productivity of postgraduate students in low- and middleincome countries. Heal Res Policy Syst, 16(1),86-87. https://health-policysystems.biomedcentral.com/articles/10.1186/s12961-018-0360-7

Pasarica, M., Bailey, M., Cendán, J. (2019). Increasing Students' Publication Productivity: Could Launching a University Scientific Journal be a Catalyst? Cureus, 11(1), e3953. https://doi.org/10.7759/cureus.3953

Quispe, A., Valentin, E., Gutierrez, A., Mares J. (2020). Serie de Redacción Científica: Estudios Trasversales. Rev. Cuerpo Med. HNAAA, 13(1), 72-77. https://doi.org/10.35434/rcmhnaaa.2020.131.626

Santa Cruz, F., \& Duran K. (2018). Significado de las prácticas tutoriales en las asesorías de tesis de los estudiantes de maestría en educación. Pag Educ, 11(2), 199-214. http://dx.doi.org/10.22235/pe.v11i2.1634

Sipahi, H., Durusoy, R., Ergin, I., Hassoy, H., Davas, A., Karababa, A. (2012). Publication rates of public health theses in international and national peer-review journals in Turkey. Iran J Public Health, 41(9), 31-5. https://www.ncbi.nlm.nih.gov/pmc/articles/PMC3494212/

Ventura-León, J. (2019). De regreso a la validez basada en el contenido. Adicciones, O(0), 1-3. https://www.adicciones.es/index.php/adicciones/article/view/1213/1038

Veytia, M., \& Contreras Y. (2019). Factores motivacionales para la investigación y los objetos virtuales de aprendizaje en estudiantes de maestría en Ciencias de la Educación. Rev Iberoam Investig Desarro Educ, 9(18), 84-101. https://doi.org/10.23913/ride.v9i18.413 